\title{
Angle of Arrival Estimation for Broadband Signals: A Comparison
}

\author{
MA Alrmah*, S Weiss*, S Redif**, S Lambotharan ${ }^{\dagger}$, JG McWhirter ${ }^{\ddagger}$ \\ * Department of Electronic and Electrical Engineering, University of Strathclyde, Glasgow, Scotland \\ ${ }^{* *}$ Electronic \& Electrical Engineering Department, European University of Lefke, Lefke, Cyprus \\ ${ }^{\dagger}$ School of Electronic, Electrical and Systems Engineering, Loughborough University, Loughborough, UK \\ ${ }^{\ddagger}$ School of Engineering, University of Cardiff, Cardiff, Wales \\ \{mohamed.alrmah,stephan.weiss\}@strath.ac.uk, sredif@eul.edu.tr, s.lambotharan@lboro.ac.uk,.mcwhirterjg@cardiff.ac.uk
}

\begin{abstract}
This paper reviews and compares three different linear algebraic signal subspace techniques for angle of arrival estimation. These include a polynomial matrix approach to multiple signal classification (MUSIC), a parameterised spatial covariance matrix approach, and an auto-focussing based version of coherent signal subspace estimation applied to MUSIC. These approaches are expressed in the framework of polynomial space-time covariance matrices and their polynomial eigenvalue decomposition, thus highlighting their commonalities and differences. Simulation results comparing the accuracy of these broadband angle of arrival estimation methods are presented.
\end{abstract}

\section{Introduction}

Powerful narrowband angle of arrival (AoA) estimation methods, such as the multiple signal classification (MUSIC) algorithm [1], are not directly applicable to the broadband case, where time delays rather than phase shift need to be considered in distinguishing between spatially separated sources. Narrowband approximations to the broadband case, such as performing the MUSIC algorithm independently in frequency bins, does not work well if sources are coherent [2] or signal frequencies do not coincide with frequency bins, leading to poor worst-case performance scenarios [3].

Dedicated broadband AoA estimation algorithms include the coherent signal subspace (CSS) method [2,4], which combines covariance matrices at different frequency bins coherently by means of focussing matrices. The focussing matrices, in their simplest form, pre-steer the data such that the signal of interest appears towards broadside, where narrowband methods suffice for estimation. Determining the focussing matrices usually requires a degree of knowledge of the scenario prior to estimation. A recent approach to auto-focussing [5] claims to overcome this problem, and is based on a bin-wise eigenvalue decomposition of covariance matrices.

Further broadband approaches include a recent parameterised spatial covariance (PSC) approach [6,7] which also pre-steers the data to then form a narrowband covariance matrix, and a subspace approach [8] derived from a polynomial eigenvalue decomposition of the space-time covariance matrix [9], which has been employed to generalise MUSIC to the broadband case.

This paper analyses the auto-focussing, parameterised spatial covariance matrix, and polynomial MUSIC approaches in the framework of polynomial space-time covariance matrices and broadband steering vectors. To achieve this, the broadband array model, and resulting steering vectors and covariance matrix are introduced in Sec. 2.. Broadband approaches to AoA estimation are outlined in Sec. 3. and, together with Sec. 4., formulated within a polynomial matrix framework. A simulation comparison between the different methods is then performed in Sec. 5., with conclusions being drawn in Sec. 6.

Notation. Matrix and vector quantities are represented by upper- and lowercase bold variables, e.g. A and a. The Hermitian transpose of $\mathbf{A}$ is denoted as $\mathbf{A}^{\mathrm{H}}$. Polynomial vectors and matrices are written as $\mathbf{a}(z)$ and $\mathbf{A}(z)$, with the parahermitian $\tilde{\mathbf{A}}(z)=\mathbf{A}^{\mathrm{H}}\left(z^{-1}\right)$. A transform pair $a[n]$ and $A(z)=\sum_{n=-\infty}^{\infty} a[n] z^{-n}$ is abbreviated as $a[n] \circ \longrightarrow A(z)$.

\section{Broadband Steering Vectors and Space-Time Covari- ance Matrix}

Below, we define a steering vector to characterise a source in a broadband scenario in Sec. 2.1, which is used to define a polynomial space-time covariance matrix in in Sec. 2.2 and its polynomial eigenvalue decomposition in Sec. 2.3.

\subsection{Broadband Steering Vector}

Given an array of $M$-element containing omnidirectional sensors located at positions $\mathbf{r}_{m}, m=1 \ldots M$, a signal vector $\mathbf{x}[n]$,

$$
\mathbf{x}[n]=\left[\begin{array}{c}
\delta\left[n-\Delta \tau_{1}\right] \\
\delta\left[n-\Delta \tau_{2}\right] \\
\vdots \\
\delta\left[n-\Delta \tau_{M}\right]
\end{array}\right] * s[n]=\mathbf{a}[n] * s[n]
$$

is collected if the array is illuminated by a source. We assume that the source signal measured at the origin is $s[n]$, with $*$ denoting convolution and delays $\Delta \tau_{m}=\frac{1}{c f_{s}} \mathbf{k}^{\mathrm{T}} \mathbf{r}_{m}, m=1 \ldots M$, 
where $c$ is the propagation speed in the medium, $f_{s}$ is the sampling rate, $\mathbf{k}$ is the normal vector of the source's wave front, and $\mathbf{k} / c$ is known as the slowness vector of the source.

The delays $\delta[n-\Delta \tau]$ are generally fractional delays [10], implementable e.g. via sinc functions sampled off their zerocrossing, $\delta[n-\Delta \tau]=\operatorname{sinc}[n-\Delta \tau]$, while $\mathbf{a}[n]$ in (1) is referred to as a broadband steering vector. The later, through the normal vector k's dependence on azimuth $\varphi$ and elevation $\vartheta$,

$$
\mathbf{k}_{\varphi, \vartheta}=\left[\begin{array}{c}
\cos \phi \sin \theta \\
\sin \phi \sin \theta \\
\cos \theta
\end{array}\right]
$$

defines the spatial characteristics of a far-field source, and will also be denoted as $\mathbf{a}_{\vartheta, \varphi}[n]$.

\subsection{Polynomial Space-Time Covariance Matrix}

In a scenario with $L$ independent far field broadband sources $s_{l}[n], l=1 \ldots L$, each characterised by a broadband steering vector $\mathbf{a}_{l}[n]$, the signal model becomes

$$
\mathbf{x}[n]=\sum_{l=1}^{L} \mathbf{a}_{l}[n] * s_{l}[n]+\mathbf{v}[n],
$$

with $\mathbf{v}[n]$ representing spatially and temporally uncorrelated noise with covariance $\mathscr{E}\left\{\mathbf{v}[n] \mathbf{v}^{\mathrm{H}}[n]\right\}=\sigma_{v}^{2} \mathbf{I}$. To capture information contained in the data vector $\mathbf{x}[n]$ requires a space-time covariance matrix $\mathbf{R}[\tau]=\mathscr{E}\left\{\mathbf{x}[n] \mathbf{x}^{\mathrm{H}}[n-\tau]\right\}$ with lag parameter $\tau$. The cross-spectral density matrix $\mathbf{R}(z)=\bullet-\circ \mathbf{R}[v]$,

$$
\mathbf{R}(z)=\sum_{l=1}^{L} \mathbf{a}(z) \mathbf{a}^{\mathrm{H}}\left(z^{-1}\right) R_{l}(z)+\sigma_{v}^{2} \mathbf{I}
$$

with $R_{l}(z)$ the power spectral density of the $l$ th source, forms a polynomial matrix.

2.3 Space-Time Covariance Matrix and Polynomial Eigenvalue Decomposition

The power spectral matrix in (4) can be decomposed to yield a polynomial EVD [9]

$$
\mathbf{R}(z)=\mathbf{Q}(z) \Lambda(z) \tilde{\mathbf{Q}}(z)=\sum_{m=0}^{M-1} \lambda_{m}(z) \mathbf{q}_{m}(z) \tilde{\mathbf{q}}_{m}(z)
$$

with paraunitary $\mathbf{Q}(z)$, i.e. $\mathbf{Q}(z) \tilde{\mathbf{Q}}(z)=\mathbf{I}$. The matrix $\Lambda(z)$ is diagonal and contains polynomial eigenvalues $\lambda_{m}(z)$, which are spectrally majorised, such that

$$
\lambda_{m}\left(e^{j \Omega}\right) \geq \lambda_{m+1}\left(e^{j \Omega}\right) \quad \forall \Omega, m=0 \ldots(M-2) .
$$

Thresholding the eigenvalues reveals the number of independent broadband sources contributing to $\mathbf{R}(z)$, and permits a distinction between signal-plus-noise and noise only subspaces

$$
\mathbf{R}(z)=\left[\mathbf{Q}_{s}(z) \mathbf{Q}_{n}(z)\right]\left[\begin{array}{cc}
\Lambda_{s}(z) & \mathbf{0} \\
\mathbf{0} & \Lambda_{n}(z)
\end{array}\right]\left[\begin{array}{l}
\mathbf{Q}_{s}^{\mathrm{H}}(z) \\
\mathbf{Q}_{n}^{\mathrm{H}}(z)
\end{array}\right]
$$

similar to a narrowband EVD [11]. Specifically, the nullspace $\tilde{\mathbf{Q}}_{n}(z)$

$$
\tilde{\mathbf{Q}}_{n}(z)=\left[\begin{array}{c}
\tilde{\mathbf{q}}_{L}(z) \\
\vdots \\
\tilde{\mathbf{q}}_{M-1}(z)
\end{array}\right]
$$

is spanned by vectors $\tilde{\mathbf{q}}_{L}(z)$ which have the same appearance as broadband steering vectors.

\section{Broadband Angle of Arrival Estimation}

In analysing broadband AoA estimation approaches, we first review the parameterised spatial covariance matrix method [6,7] in Sec. 3.1, followed by an auto-focussing approach [5] to coherent signal subspace-based estimation in Sec. 3.2 and polynomial MUSIC [8] in Sec. 3.3.

\subsection{Parameterised Spatial Correlation (PSC) Matrix Method}

The idea of the broadband AoA estimation method in [6,7] is based on testing the zero-lag coherence of a spatial correlation matrix calculated from appropriately pre-steered array data. Knowing the array configuration, a broadband steering vector can be defined for a specific AoA represented by azimuth $\varphi$ and elevation $\vartheta$, and pre-steering can be accomplished by a matched broadband steering vector. The covariance matrix of the pre-steered data is given by

$$
\begin{aligned}
\mathbf{R}_{\varphi, \vartheta} & =\mathscr{E}\left\{\mathbf{y}_{\varphi, \vartheta}[n] \mathbf{y}_{\varphi, \vartheta}^{\mathrm{H}}[n]\right\} \\
\mathbf{y}_{\varphi, \vartheta}[n] & =\left[\begin{array}{c}
x\left[n-\Delta \tau_{0}(\varphi, \vartheta)\right] \\
\vdots \\
x\left[n-\Delta \tau_{M-1}(\varphi, \vartheta)\right]
\end{array}\right]=\Gamma_{\varphi, \vartheta}[n] * \mathbf{x}[n]
\end{aligned}
$$

with the delay $\Delta \tau_{m}(\varphi, \vartheta)$ calculated akin to Sec. 2.1, and the diagonal pre-steering system

$$
\Gamma_{\varphi, \vartheta}[n]=\operatorname{diag}\left\{\delta\left[n-\Delta \tau_{0}(\vartheta)\right] \ldots \delta\left[n-\Delta \tau_{M-1}(\vartheta)\right]\right\}
$$

The proposed method then evaluates the maximum eigenvalue of $\mathbf{R}_{\varphi, \vartheta}$ in (9) for a range of angles $\{\varphi, \vartheta\}$, with the best match indicated by $\left\{\varphi_{\text {opt }}, \vartheta_{\text {opt }}\right\}=\arg \max _{\varphi, \vartheta}\left\{\max _{i} \lambda_{i}\left(\mathbf{R}_{\varphi, \vartheta}\right)\right\}$, where $\lambda_{i}\left(\mathbf{R}_{\varphi, \vartheta}\right)$ denotes the $i$ th eigenvalue of $\mathbf{R}_{\varphi, \vartheta}$.

In terms of the space time covariance matrix and broadband steering vectors defined in Sec. 2., the problem can formulated as

$$
\left\{\varphi_{\text {opt }}, \vartheta_{\text {opt }}\right\}=\underset{\varphi, \vartheta}{\arg \max _{i}}\left\{\max _{i} \lambda_{i}\left(\hat{\mathbf{R}}_{\varphi, \vartheta}[0]\right)\right\}
$$

with $\hat{\mathbf{R}}_{\varphi, \vartheta}[0]$ being the evaluation for lag zero of the space-time covariance matrix $\hat{\mathbf{R}}_{\varphi, \vartheta}[\tau] \circ \longrightarrow \bullet \hat{\mathbf{R}}_{\varphi, \vartheta}(z)$

$$
\hat{\mathbf{R}}_{\varphi, \vartheta}(z)=\operatorname{diag}\left\{\mathbf{a}_{\varphi, \vartheta}(z)\right\} \mathbf{R}(z) \operatorname{diag}\left\{\tilde{\mathbf{a}}_{\varphi, \vartheta}(z)\right\}
$$

of the pre-steered data. 


\subsection{Coherent Signal Subspace Method}

In the coherent signal subspace approach [2,4], covariance matrices are calculated in a number of frequency bins, which are then coherently combined such that their signal subspaces align into one single scalar-valued correlation matrix to which narrowband high resolution AoA techniques such as MUSIC can be applied. The coherence across different frequency bins is created by a frequency-dependent and unitary focussing matrix $\mathbf{T}\left(e^{j \Omega}\right)$, such that

$$
\mathbf{R}_{\mathrm{coh}}=\sum_{i=n}^{N-1} \alpha_{n} \mathbf{T}\left(e^{j \Omega_{n}}\right) \mathbf{R}\left(e^{j \Omega_{n}}\right) \mathbf{T}^{\mathrm{H}}\left(e^{j \Omega}\right)
$$

where $\alpha_{n}$ a weighting for maximum ratio combination of its coherently rotated contributions. The calculation of focussing matrices in the context of coherent signal subspace methods can be based on approximate knowledge of the AoA of the source of interest, or be obtained numerically by a best fit of a rotated $\mathbf{T}\left(e^{j \Omega_{n}}\right)$ to a reference $\mathbf{T}\left(e^{j \Omega_{o}}\right)$, whereby the rotation forms the focussing matrix.

A recent auto-focussing method in [5] calculates, based on a reference frequency $\Omega_{0}$, an EVD of the appropriate frequencybin covariance matrix $\mathbf{R}\left(e^{j \Omega_{0}}\right)$,

$$
\Lambda_{o}=\mathbf{Q}_{0}^{\mathrm{H}} \mathbf{R}\left(e^{j \Omega_{0}}\right) \mathbf{Q}_{0}
$$

Extracting the modal matrix for frequency bin $k, k=0 \ldots(K-$ $1)$, the auto-focussing matrix is constructed according to

$$
\mathbf{T}\left(e^{j \Omega_{k}}\right)=\mathbf{Q}_{0} \mathbf{Q}^{\mathrm{H}}\left(e^{j \Omega_{k}}\right)
$$

Therefore, the coherent covariance matrix in (14) can be diagonalised by $\mathbf{Q}_{0}$ to provide

$$
\Lambda_{\mathrm{coh}}=\mathbf{Q}_{0}^{\mathrm{H}} \mathbf{R}_{\mathrm{coh}} \mathbf{Q}_{0}=\operatorname{diag}\left\{\begin{array}{llll}
\lambda_{1} & \lambda_{2} & \ldots & \lambda_{M}
\end{array}\right\},
$$

with $\lambda_{m}, m=1 \ldots M$ the eigenvalues of $\mathbf{R}_{\mathrm{coh}}$ in (14). If the eigenvalues $\mathbf{R}_{\text {coh }}$ reveal $R$ linearly independent sources, then the last $M-R$ columns of $\mathbf{Q}_{0}=\left[\mathbf{Q}_{0, \mathrm{~s}} \mathbf{Q}_{0, \mathrm{~s}}^{\perp}\right]$ contained in $\mathbf{Q}_{0, \mathrm{~s}}^{\perp} \in \mathbb{C}^{M \times(M-R)}$ span the noise-only subspace of the coherent covariance matrix.

The standard narrowband MUSIC algorithm [1] can be applied to $\mathbf{R}_{\text {coh }}$ by probing its noise-only subspace $\mathbf{Q}_{0, \mathrm{~s}}^{\perp}$ with a set of narrowband steering vectors at the reference frequency $\Omega_{0}$. Based on the transform domain broadband steering vector $\mathbf{a}_{\varphi, \vartheta}(z)$ at the reference frequency $\Omega_{0}$, the MUSIC spectrum for auto-focussing (AF)

$$
\begin{aligned}
S_{\mathrm{AF}}(\varphi, \vartheta) & =\left\|\mathbf{Q}_{0, \mathrm{~s}}^{\perp} \mathbf{a}_{\varphi, \vartheta}\left(e^{j \Omega_{0}}\right)\right\|_{2}^{-2} \\
& =\frac{1}{\mathbf{a}_{\varphi, \vartheta}^{\mathrm{H}}\left(e^{j \Omega_{0}}\right) \mathbf{Q}_{0, \mathrm{~s}}^{\perp, \mathrm{H}} \mathbf{Q}_{0, \mathrm{~s}}^{\perp} \mathbf{a}_{\varphi, \vartheta}\left(e^{j \Omega_{0}}\right)}
\end{aligned}
$$

is obtained.

\subsection{Polynomial MUSIC Algorithm}

Generalising the concept of the narrowband MUSIC algorithm by using the polynomial EVD in (7), the idea is to probe the broadband noise-only subspace with broadband steering vectors, generalised quantity

$$
\Gamma_{\varphi, \vartheta}(z)=\tilde{\mathbf{a}}_{\varphi, \vartheta}(z) \mathbf{Q}_{n}(z) \tilde{\mathbf{Q}}_{n}(z) \mathbf{a}_{\varphi, \vartheta}(z)
$$

Noting that (3.3) represents a power spectral density rather than a norm measuring the vicinity of $\mathbf{a}_{\varphi, \vartheta}(z)$ to the nullspace of $\tilde{\mathbf{Q}}_{n}(z)$, two versions of the a polynomial MUSIC (PMUSIC) algorithm were proposed in [8], which are outlined below.

Spatial P-MUSIC. The energy contained in the signal vector $\tilde{\mathbf{Q}}_{n}(z) \mathbf{a}_{\varphi, \vartheta}(z)$ is related to the zero lag term $\gamma_{\varphi, \vartheta}[0]$ of the autocorrelation-type sequence $\gamma_{\varphi, \vartheta}[\tau] \circ \longrightarrow \Gamma_{\varphi, \vartheta}(z)$. This measure is only dependent on the angle of arrival $\vartheta$, and collects all energy across the spectrum. Instead of searching for the steering vectors providing minimum energy, the reciprocal

$$
S_{P S}(\varphi, \vartheta)=\frac{1}{\gamma_{\varphi, \vartheta}[0]}
$$

is maximised by the angle of arrival characterised by the azimuth/elevation pair $\{\varphi, \vartheta\}$ of signal sources.

Spatio-Spectral P-MUSIC. With (3.3) describing a power spectral density, spectral clues can be exploited in addition to the spatial information extracted by (19). Therefore in addition to spatial localisation of sources,

$$
S_{P S S}(\varphi, \vartheta, \Omega)=\left(\sum_{\tau=-\infty}^{\infty} \gamma_{\varphi, \vartheta}[\tau] e^{-j \Omega \tau}\right)^{-1}
$$

can determine over which frequency range sources in the direction defined by the steering vector $\mathbf{a}_{\varphi, \vartheta}(z)$ are active. PSSMUSIC was introduced in [8], but will be omitted from the comparison below, since the benchmark method only retrieves AoA information.

\section{Analytical Comparison}

The auto-focussing approach to coherent signal subspace estimation is linked to the polynomial EVD of the space-time covariance matrix in Sec. 4.1, while Secs. 4.2 and 4.3 relate the auto-focussing approximation of coherent signal subspace MUSIC to the polynomial PSS- and PS-MUSIC methods in [8].

\subsection{Auto-Focussing Matrices via Polynomial Eigenvalue Decomposition}

With the modal matrix $\mathbf{Q}_{0}$ in (16) obtained at the reference frequency $\Omega_{0}$ via $\operatorname{EVD}$ of $\mathbf{R}\left(e^{j \Omega_{0}}\right)$, the focussing matrix can be formulated as a paraunitary matrix $\left.\mathbf{T}(z)\right|_{z=e^{j \Omega}}=\mathbf{Q}_{0} \mathbf{Q}^{\mathrm{H}}\left(e^{j \Omega}\right)$. 
Replacing the summation over frequency bins in (14) by the integration over the Fourier transform (i.e. $K \rightarrow \infty$ ) leads to

$$
\begin{aligned}
\mathbf{R}_{\mathrm{coh}} & \approx \frac{1}{2 \pi} \oint\{\mathbf{T}(z) \mathbf{R}(z) \tilde{\mathbf{T}}(z)\}_{z=e^{j \Omega}} d \Omega \\
& =\mathbf{Q}_{0} \frac{1}{2 \pi} \oint\{\tilde{\mathbf{Q}}(z) \mathbf{R}(z) \mathbf{Q}(z)\}_{z=e^{j \Omega}} d \Omega \mathbf{Q}_{0}^{\mathrm{H}} .
\end{aligned}
$$

Since the paraunitary matrix $\mathbf{Q}(z)$ diagonalises $\mathbf{R}(z)$, the argument under the integral is the polynomial EVD in (5), resulting in a diagonal matrix of power spectral densities, $\frac{1}{2 \pi} \oint \Gamma\left(e^{j \Omega}\right) d \Omega=\Gamma[0]$, where $\Gamma[0]$ is the evaluation of $\Gamma[\tau] \circ \longrightarrow \Gamma(z)$ for zero lag. Therefore

$$
\mathbf{R}_{\mathrm{coh}} \approx \mathbf{Q}_{0} \Gamma[0] \mathbf{Q}_{0}^{\mathrm{H}}=\mathbf{Q}_{0}\left[\begin{array}{ccc}
\sigma_{1}^{2} & & \\
& \ddots & \\
& & \sigma_{M}^{2}
\end{array}\right] \mathbf{Q}_{0}^{\mathrm{H}}
$$

represents the coherent covariance matrix of the auto-focussing approach in terms of the polynomial EVD of the cross spectral density matrix.

Given that the DFT in (14) is a sufficiently accurate representation of the Fourier transform formulation in (21), then (17) and (23) are equivalent with $\Lambda=\Gamma[0]$. Further, the PEVD of the CSD matrix provides a paraunitary $\mathbf{Q}(z)$ that leads to an autofocussing matrix $\mathbf{Q}_{0} \tilde{\mathbf{Q}}(z)$ which is continuous in frequency.

\subsection{Relation between Auto-Focussing MUSIC and PSS- MUSIC}

Provided that the estimation of the number of linearly independent sources, $R$, is the same for the auto-focussing approach of CSS and from (23) of the polynomial approach, then with $\mathbf{Q}_{0}$ being the evaluation of the paraunitary $\mathbf{Q}(z)$ at the reference frequency $\Omega_{0}$, i.e. $\mathbf{Q}_{0}=\left.\mathbf{Q}(z)\right|_{z=e^{j \Omega_{0}}}$, it follows that

$$
S_{\mathrm{CSS}}(\varphi, \vartheta)=\left.S_{\mathrm{PSS}}\left(\varphi, \vartheta, e^{j \Omega}\right)\right|_{\Omega=\Omega_{0}}
$$

Therefore, the auto-focussing approach to coherent signal subspace MUSIC estimation is equivalent to evaluating the polynomial spatio-spectral MUSIC spectrum at the reference frequency $\Omega_{0}$.

To obtain the same spatio-spectral characterisation of the array data as provided by PSS-MUSIC with the CSS approach, a sequence of different modal matrices $\mathbf{Q}_{0}$ at different reference frequencies $\Omega_{0}$ could be calculated, for all of which (18) is evaluated.

\subsection{Relation between Auto-Focussing MUSIC and PS- MUSIC}

Noting that in (19),

$$
\gamma_{\varphi, \vartheta}[0]=\frac{1}{2 \pi} \oint_{0}^{2 \pi} \Gamma_{\varphi, \vartheta}\left(e^{j \Omega}\right) d \Omega
$$

if the integral can be approximated by a sum over discrete frequency bins, i.e.

$$
\gamma \approx \frac{1}{K} \sum_{k=0}^{K-1} \mathbf{a}_{\varphi, \vartheta}^{\mathrm{H}}\left(e^{j \Omega_{k}}\right) \mathbf{Q}_{\mathrm{s}}^{\perp, \mathrm{H}}\left(e^{\Omega_{k}}\right) \mathbf{Q}_{\mathrm{s}}^{\perp}\left(e^{j \Omega_{k}}\right) \mathbf{a}_{\varphi, \vartheta}\left(e^{j \Omega_{k}}\right),
$$

then (26) is the summation over the denominator terms of (18) for all possible reference frequencies $\Omega_{k}$ with $\Omega_{k}=\frac{2 \pi}{K} k$, $k=0 \ldots(K-1)$. The PS-MUSIC denominator in the above approximation also appears similar to the incoherent MUSIC approach stated in [12]; however, here the paraunitary matrix $\mathbf{Q}(z)$ that feeds into (26) has been demonstrated in (23) to coherent combine the spatio-temporal covariance matrix in the auto-focussing sense.

\section{Simulations and Numerical Results}

Below, we illustrate the analysis of the above broadband AoA estimation algorithms in two examples.

\subsection{Example 1 - Single Source Case}

To highlight the connection between the auto-focussing approach and PSS-MUSIC, we first consider a simple toy problem with a single source in a noise-free environment, where the space-time covariance matrix and its ideal decomposition are known. Here, a single broadband source emits an uncorrelated Gaussian signal, which is captured by an $M=4$ element linear array with equispaced sensors that sample critically in both time and space. The broadband steering vector of this source is

$$
\mathbf{a}_{1}(z)=\frac{1}{\sqrt{M}}\left[\begin{array}{llll}
1 & z^{-1} & \ldots & z^{-M+1}
\end{array}\right]^{\mathrm{T}}
$$

such that the space-time covariance matrix is given by

$$
\mathbf{R}_{1}(z)=\left[\begin{array}{cccc}
1 & z^{1} & \ldots & z^{M-1} \\
z^{-1} & 1 & & \vdots \\
\vdots & & \ddots & \vdots \\
z^{-M+1} & \ldots & \ldots & 1
\end{array}\right] .
$$

Because $\mathbf{R}_{1}(z)$ is rank one, a manifold of diagonalising polynomial decompositions exists, with one possibility being

$$
\mathbf{Q}(z)=\operatorname{diag}\left\{1 z^{-1} \ldots z^{-M+1}\right\} \mathbf{T}_{\mathrm{DFT}},
$$

where $\mathbf{T}_{\mathrm{DFT}}$ is an $M$-point DFT matrix normalised by $\sqrt{M}$ such that $\mathbf{T}_{\mathrm{DFT}}$ is unitary. Based on PSS-MUSIC in (??) using a sampled sinc function, truncated to order 200, to approximate broadband steering vectors, the spectrum in Fig. 1 emerges. Normalised to a maximum value of unity, the AoA of the endfire source is identified. In line with broadband arrays, at lower frequencies the fixed aperture degrades the spatial resolution, with no ability to discern sources at DC.

For the auto-focussing approach, at a given reference frequency $\Omega_{0}, \mathbf{R}_{\mathrm{coh}, \Omega_{0}}=\left.\mathbf{R}(z)\right|_{z=e^{j \Omega_{0}}}$ and $\Lambda_{\mathrm{coh}, \Omega_{0}}=\operatorname{diag}\{1,0 \cdots 0\}$. 


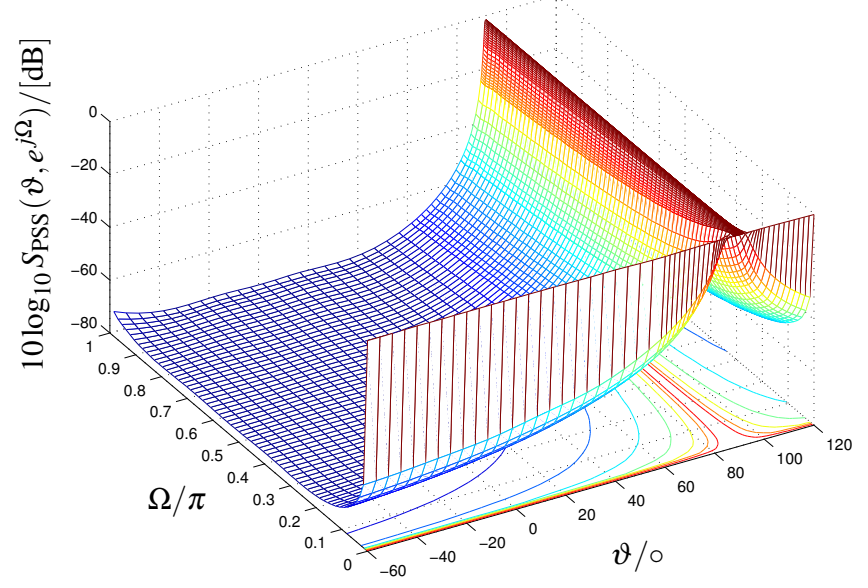

Figure 1. PSS-MUSIC spectrum for a single source at end-fire position, $\vartheta=90^{\circ}$

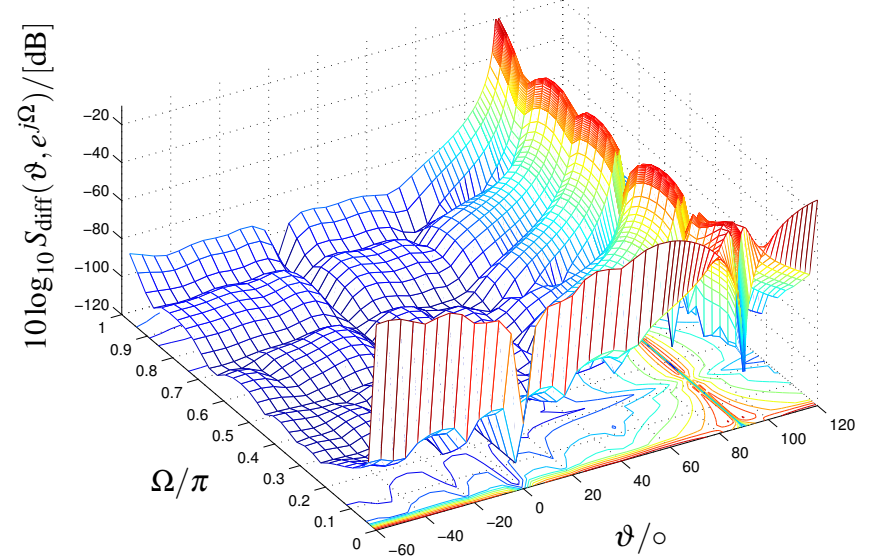

Figure 2. Difference between the PSS- and auto-focussing (AF) based MUSIC spectra for a single source at $\vartheta=90^{\circ}$.

Evaluating the narrowband MUSIC algorithm using narrowband steering vectors at $\Omega_{0}$ for a range of $K=64$ discrete equispaced reference frequencies $\Omega_{0}$, and the nullspace $\mathbf{Q}_{\mathrm{s}}^{\perp}\left(e^{j \Omega_{0}}\right)$ derived from the EVD of $\mathbf{R}_{\mathrm{coh}, \Omega_{0}}$, a MUSIC spectrum similar to Fig. 1 emerges.

The difference between the PSS- and AF-MUSIC spectra, $S_{\text {diff }}\left(\vartheta, e^{j \Omega}\right)=\left|S_{\mathrm{PSS}}\left(\vartheta, e^{j \Omega}\right)-S_{\mathrm{AF}}(\vartheta, \Omega)\right|$, is plotted in Fig.2, with an error below $-10 \mathrm{~dB}$. The error is largest where the MUSIC spectrum is numerically most sensitive, i.e. towards the source at $\vartheta=90^{\circ}$, and for DC, $\Omega=0$. The error can be attributed to the inaccuracies of the truncated sinc functions to implement fractional delays for the broadband steering vector, while narrowband steering vectors required for CSSMUSIC can always be accurately represented. Note that for the trivial broadband steering vector towards broadside, $\mathbf{a}_{0^{\circ}}(z)=\frac{1}{M}\left[\begin{array}{llll}1 & 1 & \ldots & 1\end{array}\right]^{\mathrm{T}}$, the error in Fig. 2 for $\vartheta=0^{\circ}$ is negligible.

For this case with a single source and known covariance matrix, the integration of the PSS-MUSIC spectrum across frequency yields the PS-spectrum, which in Fig. 3 is shown for both the correct PEVD and its iterative approximate solution using the second order sequential best rotation algorithm (SBR2) [9]. The significant degradation is due to the sensitivity
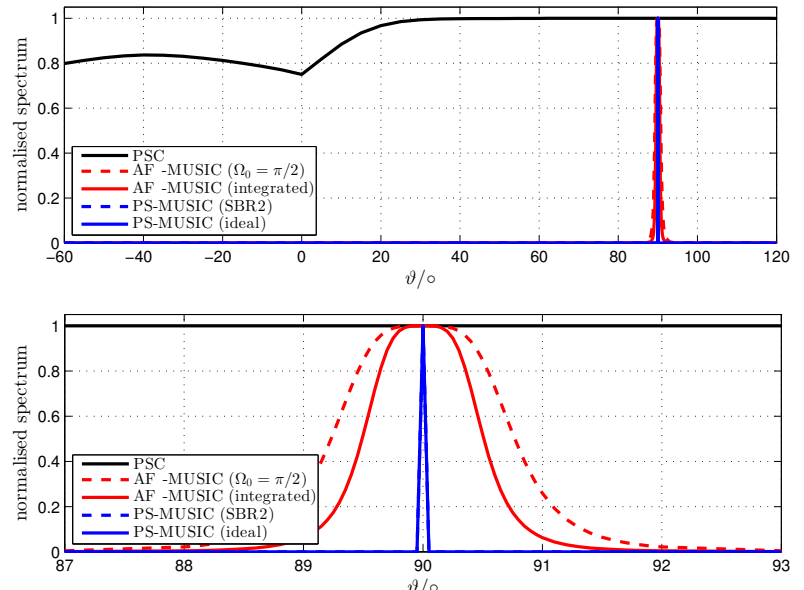

Figure 3. Comparison between PSC, AF-MUSIC (i) evaluated at the centre frequency $\Omega_{0}=\frac{\pi}{2}$ and (ii) integrated according to (26), and PS-MUSIC (iii) with SBR2-estimated and (iv) ideal PEVD; the bottom graph shows a detail of the overall response in the top figure.

of the MUSIC algorithm to small modifications of the denominator when close to zero. It can be seen that the difference to the integrated CS-MUSIC is very small. As a benchmark, the parameterised spatial covariance matrix approach [6,7] is also plotted in Fig. 3. Unlike the MUSIC approaches, PSC does not display an inverse value, and hence will not exhibit the same sharp peaks and at least appear to possess a much lower resolution in comparison.

\subsection{Example 2 - Dual Source Case}

In addition to a source at end-fire, a second independent source is assumed to illuminate from broadside, $\vartheta=0^{\circ}$. Assumed to be temporally uncorrelated with unit variance, its broadband steering vector is

$$
\mathbf{a}_{2}(z)=\frac{1}{\sqrt{M}}\left[\begin{array}{llll}
1 & 1 & \ldots & 1
\end{array}\right]^{\mathrm{T}},
$$

leading to a non-polynomial space-time covariance matrix with unity for all entries. With a relative signal strength of $0 \mathrm{~dB}$ for each source, additional i.i.d. noise contaminates all sensors at a variance of $-20 \mathrm{~dB}$, giving an SINR of $-23 \mathrm{~dB}$. Therefore the overall space-time covariance is

$$
\mathbf{R}_{2}(z)=\mathbf{R}_{1}(z)+\frac{1}{M}\left[\begin{array}{cccc}
1 & 1 & \ldots & 1 \\
\vdots & & \ddots & \vdots \\
1 & 1 & \ldots & 1
\end{array}\right]+\sigma_{v}^{2} \mathbf{I}
$$

with no obvious PEVD as for $\mathbf{R}_{1}(z)$ in (29).

The PSS-MUSIC spectrum based on an SBR2-approximation of the PEVD is shown in Fig. 4, which offers a similar quality to the AF-MUSIC spectrum scanned for different reference frequencies in Fig. 5. The degradation of the PSS-MUSIC spectrum's surface compared to the AF-approach, particularly at peak values, is due to the SBR2 algorithm's approximate nature. 


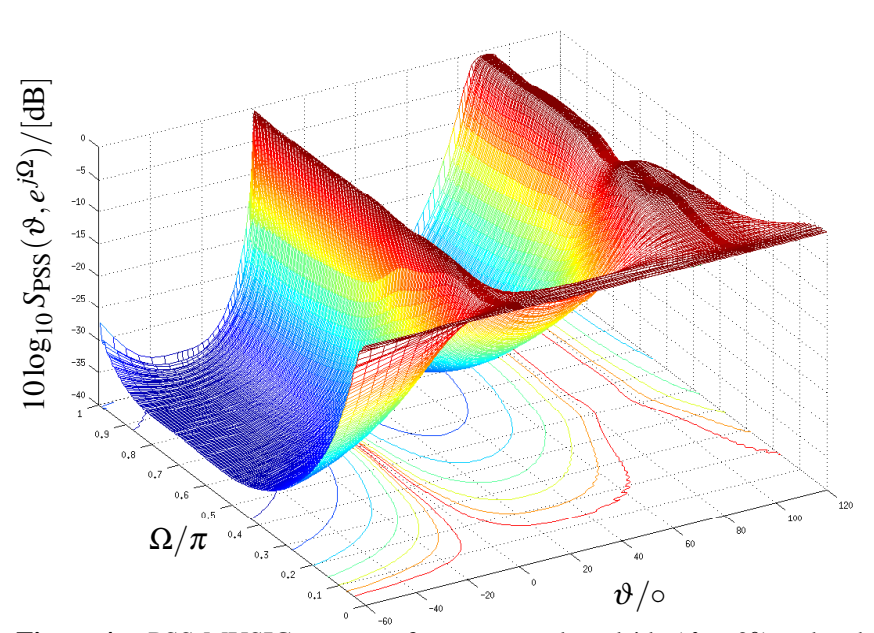

Figure 4. PSS-MUSIC spectrum for sources at broadside $\left(\vartheta=0^{\circ}\right)$ and endfire $\left(19=90^{\circ}\right)$.

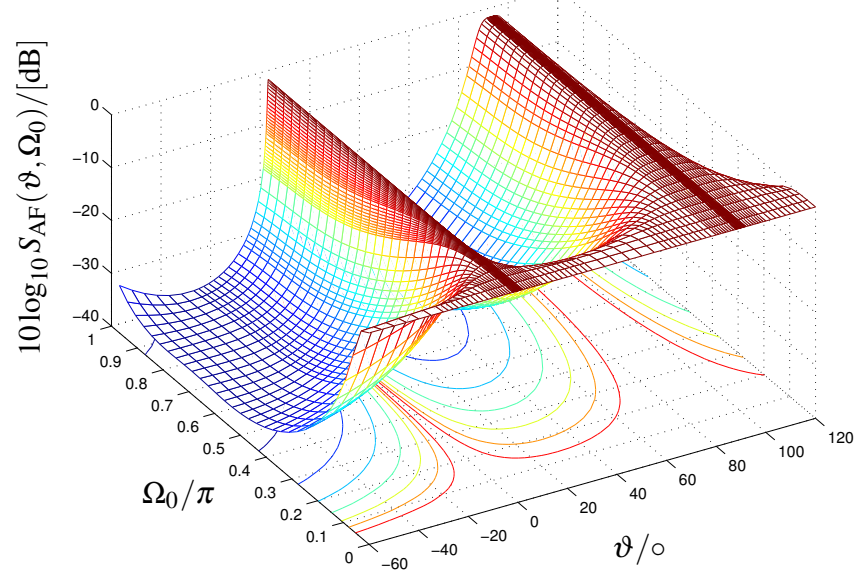

Figure 5. AF-MUSIC spectrum evaluated over a range of reference frequencies $\Omega_{0}$ for the scenario in Fig. 4 .

The spatial-only spectra for the dual source case in Fig. 6 illustrate, that PSC is only able to resolve a single source, while AF- and PS-MUSIC approaches correctly detect the sources, with AF offering a higher resolution due to the SBR2approximation of the ideal PEVD for the PS-MUSIC case.

\section{Conclusions}

Three broadband angle of arrival estimation algorithms have been reviewed and analysed, including a parameterised spatial covariance matrix approach, which by definition can only resolve the strongest source, an auto-focussing approximation of the coherent signal subspace method, where the broadband problem is transformed to yield a narrowband one, and a polynomial MUSIC approach. The algorithms have been cast in the framework of broadband steering vectors and broadband space-time covariance matrices, and the links in particular between the auto-focussing approach and polynomial MUSIC have been stated, which generally rely on the transition from a discrete evaluation of frequency bins to a continuous spectrum. In simulations, AF- and polynomial MUSIC approaches have proven very similar, with differences arising from the iterative approximation of the polynomial EVD. However, a

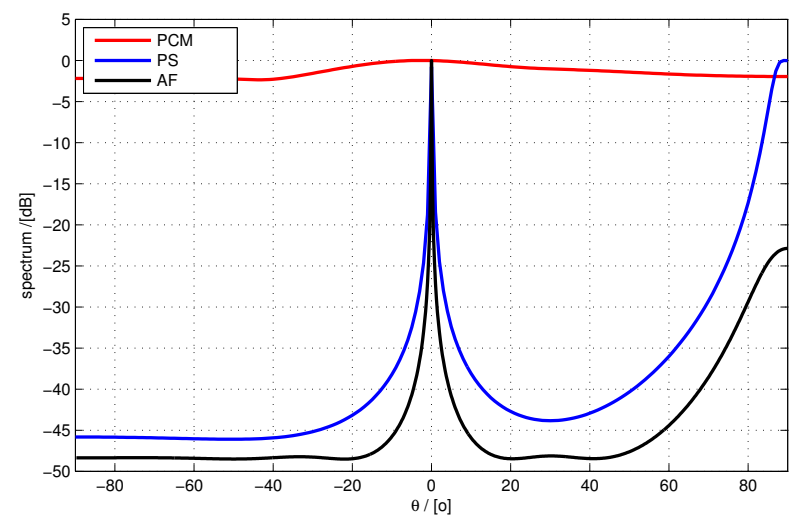

Figure 6. PSC compared to PS- and AF-spectra, normalised to unity, for the dual source case.

natural advantage of the polynomial MUSIC approach is its potential resolution of both angle of arrival and frequency.

\section{References}

[1] R. O. Schmidt, "Multiple emitter location and signal parameter estimation," IEEE Transactions on Antennas and Propagation, vol. 34, no. 3, pp. 276-280, March 1986.

[2] H. Wang and M. Kaveh, "Coherent signal-subspace processing for the detection and estimation of angles of arrival of multiple wide-band sources," IEEE Transactions on Acoustics, Speech and Signal Processing, vol. 33, no. 4, pp. 823-831, Aug 1985.

[3] S. Weiss and I. K. Proudler, "Comparing Efficient Broadband Beamforming Architectures and Their Performance TradeOffs," in 14th International Conference on Digital Signal Processing, vol. I, Santorini, Greece, July 1-3 2002, pp. 417-422.

[4] H. Hung and M. Kaveh, "Focussing matrices for coherent signal-subspace processing," IEEE Transactions on Acoustics, Speech and Signal Processing, vol. 36, no. 8, pp. 1272-1281, Aug. 1988.

[5] P. Pal and P. P. Vaidyanathan, "A novel autofocusing approach for estimating directions-of-arrival of wideband signals," in Forty-Third Asilomar Conference on Signals, Systems and Computers, Pacific Grove, CA, November 2009, pp. 16631667.

[6] J. Dmochowski, J. Benesty, and S. Affes, "Direction of arrival estimation using the parameterized spatial correlation matrix," IEEE Transactions on Audio, Speech, and Language Processing, vol. 15, no. 4, pp. 1327-1339, May 2007.

[7] M. Souden, J. Benesty, and S. Affes, "Broadband source localization from an eigenanalysis perspective," IEEE Transactions on Audio, Speech, and Language Processing, vol. 18, no. 6, pp. 1575-1587, August 2010.

[8] M. Alrmah, S. Weiss, and S. Lambotharan, "An extension of the music algorithm to broadband scenarios using polynomial eigenvalue decomposition," in 19th European Signal Processing Conference, Barcelona, Spain, August 2011, pp. 629-633.

[9] J. G. McWhirter, P. D. Baxter, T. Cooper, S. Redif, and J. Foster, "An EVD Algorithm for Para-Hermitian Polynomial Matrices," IEEE Transactions on Signal Processing, vol. 55, no. 5, pp. 2158-2169, May 2007.

[10] T. I. Laakso, V. Välimäki, M. Karjalainen, and U. K. Laine, "Splitting the Unit Delay," IEEE Signal Processing Magazine, vol. 13, no. 1, pp. 30-60, January 1996.

[11] G. H. Golub and C. F. Van Loan, Matrix Computations, 3rd ed. Baltimore, Maryland: John Hopkins University Press, 1996.

[12] Y.-S. Yoon, L. Kaplan, and J. McClellan, "TOPS: new DOA estimator for wideband signals," IEEE Transactions on Signal Processing, vol. 54, no. 6, pp. 1977-1989, June 2006. 